\title{
Spider mites escape bacterial infection by avoiding contaminated food
}

Flore Zélé ${ }^{1}$, Gonçalo Santos-Matos* ${ }^{1,2}$, Alexandre Figueiredo ${ }^{1,3,4}$, Cátia Eira ${ }^{1}$, Catarina Pinto ${ }^{1}$, Telma Laurentino ${ }^{5}$, Élio Sucena**2,6 and Sara Magalhães**1,6

*Joint first authors

**Joint last authors

\# Corresponding author: snmagalhaes@fc.ul.pt

${ }^{1}$ Centre for Ecology, Evolution and Environmental Changes (cE3c), Faculdade de Ciências da Universidade de Lisboa, Edificio C2, 3ํ Piso Campo Grande, 1749-016 Lisboa, Portugal

${ }^{2}$ Instituto Gulbenkian de Ciência, Apartado 14, 2781-901 Oeiras, Portugal

${ }^{3}$ Department for Plant and Microbial Biology, University of Zurich, Winterthurerstrasse 190, 8057 Zürich, Switzerland

${ }^{4}$ Department of Evolutionary Biology and Environmental Studies, University of Zurich, Winterthurerstrasse 190, 8057 Zürich, Switzerland

${ }^{5}$ Zoological Institute, University of Basel, Vesalgasse 1, 4051 Basel, Switzerland

6 Departamento de Biologia Animal, Faculdade de Ciências da Universidade de Lisboa, Campo Grande, Edifício C2, 1749-016 Lisboa, Portugal

\section{AUTHORS' CONTRIBUTIONS}

Experimental conception and design: $F Z, G M, E S, S M$; acquisition of data: $G M, A F, C E, C P, T L$; statistical analyses: FZ; paper writing: FZ, GM, SM, with input from all authors. Funding: ES, SM. All authors have read and approved the final version of the manuscript. 


\section{Abstract}

To fight infection, arthropods rely on the deployment of an innate immune response but also upon physical/chemical barriers and avoidance behaviours. However, most studies focus on immunity, with other defensive mechanisms being relatively overlooked.

We have previously shown that the spider mite Tetranychus urticae does not mount an induced immune response towards systemic bacterial infections, entailing very high mortality rates. Therefore, we hypothesized that other defence mechanisms may be operating to minimize infection risk. Here, we test (a) if spider mites are also highly susceptible to other infection routes - spraying and feeding - and (b) if they display avoidance behaviours towards infected food. Individuals sprayed with or fed on Escherichia coli or Pseudomonas putida survived less than the control, pointing to a deficient capacity of the gut epithelium, and possibly of the cuticle, to contain bacteria. Additionally, we found that spider mites prefer uninfected food to food contaminated with bacteria, a choice that probably does not rely on olfactory cues.

Our results suggest that spider mites may rely mostly on avoidance behaviours to minimize bacterial infection and highlight the multi-layered nature of immune strategies present in arthropods.

\section{Keywords}

Parasitism, immunity, behavioural avoidance, Tetranychus urticae 


\section{INTRODUCTION}

Arthropods are often attacked by pathogens, leading to severe fitness costs. In response, arthropods have evolved several mechanisms to counter infection, ranging from behavioural avoidance to the deployment of an immune response. While a large body of literature concerns the immune response of arthropods to bacterial infection (Schmid-Hempel 2005; Lemaitre and Hoffmann 2007; Graham et al. 2011), these were found to be inefficient in fighting infection in both aphids (Altincicek et al. 2008; Gerardo et al. 2010; but see Laughton et al. 2016; Parker et al. 2017) and spider mites (Santos-Matos et al. 2017) compared to other invertebrate studied to date.

Systemic immunity operates once a pathogen is inside the body of a host. However, pathogens must cross a series of barriers before entering their hosts (Hall et al. 2017). Indeed, many pathogens are ingested by their host but need to bypass the gut epithelium to cause infection, point at which most pathogens are contained (Kuraishi et al. 2011; Martins et al. 2013). Such strategies are not detected by classical immunological studies, which investigate responses upon injection of the parasite into the body cavity of the host. Moreover, ingestion of potential pathogenic agents may be controlled through host behavioural responses (Clayton 1991). However, despite their importance as a first line of defence against parasites (Hart 1990; Hart 1994) and their predicted strong effect on hostparasite dynamics (Eakin et al. 2015), behavioural strategies to avoid infection remain much less studied than 'classical' immune responses (reviewed by Parker et al. 2011), or than behavioural strategies to avoid predation (reviewed by Buck et al. 2018). Moreover, avoidance behaviour is likely the most cost-effective strategy employed by free-living animals to maintain fitness in the face of parasite threat, as compared to resistance and tolerance (Curtis 2014). Indeed, many behavioral strategies allowing animals to avoid exposure to parasites, and thus the likelihood of infection, have been described (for reviews, see Buck et al. 2018; Cremer et al. 2018; Curtis 2014; Moore 2002). For example, hosts may detect parasites and avoid them directly, such as aphids dropping from plants to escape parasitoid wasps (Fill et al. 2012); or avoid habitats harbouring pathogens, such as the rainbow trout avoiding shelters where eye fluke cercariae are present (Karvonen et al. 2004). Hosts may also avoid contact with infected conspecifics, such as bullfrog tadpoles when facing yeast infection (Kiesecker et al. 1999). Finally, hosts may avoid ingesting infected food, 
such as gypsy moth larvae that avoid feeding on foliage contaminated with cadavers of virusinfected conspecific larvae (Parker et al. 2010). Some organisms are also able to avoid different sources of infection, such as adult seven-spot ladybirds, which avoid the fungus Beauveria bassiana on leaf surfaces, in soil and in mycosed conspecifics cadavers (Ormond et al. 2011). However, some organisms lack pathogen avoidance behaviours (Mnyone et al. 2010); or may even be attracted toward infected conspecifics (Bouwman and Hawley 2010; Cornet et al. 2013).

We have previously shown that most genes acting in Drosophila immunity, which are generally conserved among arthropods, are absent in the spider mite Tetranychus urticae (Grbic et al. 2011). Furthermore, we showed that spider mites do not mount an immune response and die upon infection by both gram-positive and gram-negative bacteria (SantosMatos et al. 2017). However, these conclusions were based upon experiments using bacterial injection, a route of infection that bypasses a series of possible barriers to infection. Here, we set out to investigate (a) whether spider mites are also susceptible to bacterial infection by spraying (i.e., topical infections and/or bacteria ingestion) or to bacteria ingestion only, (b) whether they are able to avoid contaminated food, and (c) whether olfactory cues play a role in such avoidance. To this aim, we used two species of bacteria: a non-pathogenic strain of Escherichia coli, commonly used in immunity studies (Lemaitre et al. 1996; Santos-Matos et al. 2017) and found on plants (Meric et al. 2013; Seo and Matthews 2012; Solomon et al. 2003; Solomon et al. 2002); and Pseudomonas putida, a bacterium commonly found on plants (Bodenhausen et al. 2013; Rastogi et al. 2012), which is pathogenic to spider mites (Aksoy et al. 2008) and other arthropods (Ateyyat et al. 2010; Ayres and Schneider 2008; Schneider and Dorn 2001) as other Pseudomonas species (Broadbent and Matteoni 1990; Bucher and Stephens 1957; Commare et al. 2002; Poinar and Poinar 1998; Thomas and Poinar 1973; Vodovar et al. 2006; Vodovar et al. 2005).

\section{Material and Methods}

\section{Spider mite populations}

All experiments were done with the two-spotted spider mite, Tetranychus urticae. For the olfactory cue experiments, we used the TuTom.tet population, originally collected from 
tomato plants in Carregado, Portugal, in August 2010 (Clemente et al. 2016). Other experiments were performed using a population derived from the London strain. This strain was originally collected from fields in the Vineland region, Ontario, Canada and was used to sequence the species genome (Grbic et al. 2011). Both populations were maintained under controlled conditions $\left(25 \pm 2^{\circ} \mathrm{C}, 16 / 8 \mathrm{~h} \mathrm{~L} / \mathrm{D}\right)$ on bean plants (Phaseolus vulgaris, variety Enana, provided by Germisem, Portugal) at the Faculty of Sciences of the University of Lisbon, since 2010. To control for age of the tested females, adult spider mite females were placed on a bean leaf inside a petri dish, where they laid eggs for 24 hours. These petri dishes were then kept under the aforementioned controlled conditions and spider mites developed into adulthood. For the experiments, we used 0-3 day-old females (since the last moult).

\section{Bacterial stocks}

Bacterial stocks of E. coli DH5 $\alpha$ (Gram negative) and P. putida (Gram negative) were retrieved from a $-80^{\circ} \mathrm{C}$ stock and plated on Petri dishes with Luria Broth (LB) 3 days before each experiment was performed. Subsequently, a colony was picked from the Petri dishes, transferred to liquid LB, grown overnight at $37^{\circ}$ for E. coli and $30^{\circ}$ for $P$. putida and diluted in LB (i.e. the same LB solution than the control: $10 \mathrm{~g} / \mathrm{L}$ tryptone; $5 \mathrm{~g} / \mathrm{L}$ Yeast Extract; $10 \mathrm{~g} / \mathrm{L} \mathrm{NaCl}$ ) to the required concentrations: optical density (OD) of 1,10 and/or 25 , measured at $600 \mathrm{~nm}$ with GENESYS ${ }^{\mathrm{TM}}$ 10S UV spectrophotometer (Thermo Scientific ${ }^{\mathrm{TM}}$ ), depending on the experiment. Although OD is a standard measurement in microbiology, the concentration of both bacteria species may differ for a same OD value (e.g. OD1 of E. coli corresponds to $8 x$ $10^{8}$ cells $/ \mathrm{ml}$, while OD1 of $P$. aeruginosa correspond to $2 \times 10^{8}$ cells $/ \mathrm{mL}$; Kim et al. 2012). Since the concentration of $P$. putida is, to our knowledge, unknown, we performed a simple test to determine its concentration relative to that of E. coli. $100 \mu \mathrm{l}$ of solution of each species (bacteria at OD1 serially diluted to 1:800000) were plated in 5 different petri dishes, and the number of Colony Forming Units (CFUs) was counted in each dish the next day. Plates inoculated with $P$. putida contained $57.8 \pm 3.2$ CFUs, while those with $E$ coli had 145.4 \pm 6.4 CFUs.

\section{Effects of bacteria spraying on spider-mite mortality and oviposition}

The effects of $E$. coli and of $P$. putida infection by spraying were tested in two separate 
experiments, each consisting of 3 independent experimental blocks. For each block, $200 T$. urticae females were individually placed on bean leaf fragments $(1.5 \mathrm{~cm}$ length and $1 \mathrm{~cm}$ width) placed on wet cotton inside Petri dishes (up to 25 leaf fragments per Petri dish). This procedure prevents spider mites from escaping the leaf fragments (individuals that fall out of the leaf fragments are drowned in the wet cotton). Subsequently, each Petri dish was sprayed three times using a sprinkler $(0.33 \mathrm{ml}$ per spatter; c.a. $1 \mathrm{ml}$ per Petri dish), at a height of $30 \mathrm{~cm}$, with bacteria at OD1, OD10, or OD25, or with LB as control (i.e., 50 females were allocated to each treatment). Subsequently, spider mites were kept in a controlled environment $\left(25 \pm 2^{\circ} \mathrm{C}, 16 / 8 \mathrm{~h} \mathrm{~L} / \mathrm{D}\right)$ for 96 hours. Survival and fecundity were recorded every 24 hours. For each bacterial species, a total of 600 mites were thus tested (50 per treatment per block).

\section{Effects of bacteria ingestion on spider-mite mortality and fecundity}

To measure spider mite mortality and fecundity upon bacterial ingestion, 40 females were placed on circular cardboard arenas (ca. $3 \mathrm{~cm}^{2}$ ) with 2 parafilm bubbles (made using a vacuum manifold attached to a vacuum pump and subsequently closed using adhesive tape). The bubbles were filled with $30 \mu \mathrm{l}$ of food consisting of a 1:1 proportion of Schneider's medium and either LB (negative control) or LB with bacteria at OD1, OD10, or OD25. A green food colouring dye was added to the mixture at a 1:4 proportion to distinguish spider mites that fed on the bubbles from those that did not (mites are relatively transparent hence the dye is easily seen inside mites). Mites were then kept in the arenas for 48 hours before scoring the number of females alive and those with dye in their gut. Females that had dye in the gut were individually placed on bean leaf fragments $(1.5 \mathrm{~cm}$ length and $1 \mathrm{~cm}$ width) over wet cotton inside Petri dishes (up to 25 leaf fragments per Petri dish) and kept in a controlled environment $\left(25 \pm 2^{\circ} \mathrm{C}, 16 / 8 \mathrm{~h}\right.$ L/D) for 96 hours during which survival and fecundity were recorded every 24 hours. As mentioned above, the effects of infection by $E$. coli and P. putida were tested in two separate experiments with an initial number of 400 mites each and consisting of 5 independent experimental blocks, with two arenas per block.

\section{Effects of volatile cues on spider mite avoidance behaviour}


To investigate whether spider mites use olfactory cues to avoid food sources contaminated with bacteria, we performed two experiments using previously established protocols (Pallini et al. 1997; Rodrigues et al. 2017). In a first experiment, we performed a two-way choice test where adult females were placed in the middle of a dumbbell-shaped arena consisting of a strip of parafilm $(2 \mathrm{~cm} \times 0.5 \mathrm{~cm})$ connecting one bean leaf disc $\left(0.64 \mathrm{~cm}^{2}\right)$ with a droplet $(10$ $\mu \mathrm{L}$ ) of LB and another with either no droplet (control), a droplet containing E. coli (OD10), or a droplet containing $P$. putida (OD10). Trials began as soon as individuals were placed in the centre of the parafilm bridge and lasted 30 minutes. The time spent by a female on either side of the arena was recorded. A test was considered invalid if an individual drowned or failed to leave the centre of the arena. A total of 66 females were tested in this experiment ( $\mathrm{n}=21$ for the control, $\mathrm{n}=22$ for $P$. putida, and $\mathrm{n}=23$ for $E$. coli).

In a second experiment, we used a Y-maze olfactometer connected to a vacuum pump with an airflow of $0.4-0.5 \mathrm{~m} / \mathrm{s}$ to test avoidance of $E$. coli (OD25) or of P. putida (OD25) by $T$. urticae females. Two types of tests were performed. In the first, we tested whether spider mites use olfactory cues to avoid bacteria in absence of the food source. Cotton soaked in LB was placed inside a syringe connected to one of the Y-maze arm, while cotton soaked in bacteria was connected to the other. In the second test, we tested whether spider mites use olfactory cues to avoid food sources contaminated with bacteria. The day before the test, very early in the morning (optimal for pathogen proliferation), bean plants (14 daysold) were covered with a transparent plastic dome inside the growing chamber $\left(25^{\circ} \mathrm{C}, 16 / 8 \mathrm{~h}\right.$ L/D) and soaked in water for 2-4 hours to increase stomatal opening (Liu et al. 2015). 24h before the test, 24 droplets of $10 \mu$ l of the bacterial suspension at OD25 or of LB (as control), were placed on two leaves of the experimental plants. The plants were then placed under a transparent plastic dome under the light in the experimental room for one day to maintain humidity (Liu et al. 2015) and to avoid communication between plants. On the day of the test, both bean plants with LB or with bacteria were placed individually in an experimental plastic box $(18.5 \times 18.8 \times 24.5 \mathrm{~cm})$ connected to each arm of the $\mathrm{Y}$-maze. For both tests, PES Sterile Membrane Filters $\left(0.2 \mu \mathrm{m}\right.$; VWR $\left.{ }^{\mathrm{TM}}\right)$ were connected to the tip of the tube connecting the syringes or the plants' boxes to the olfactometer to ensure that bacteria did not contact with the olfactometer arms and that only olfactory cues could be perceived. One-day old adult females were then placed at the end of the Y-maze and allowed to move along the 
central wire of the olfactometer, without touching the glass walls. Each female was tested individually and a choice was considered valid when the mite entered the last $1 / 3$ of one of the olfactometer arms within five minutes. To avoid confounding effects, bacteria were swapped from one olfactometer arm to the other every 5 valid choices. For each test, a total of 80 valid choices were obtained within four experimental blocks ( 20 choices per block), each experimental block corresponding to a different day.

\section{Statistical analyses}

Analyses were carried out using the R statistical package (v. 3.3.2). The different statistical models built to analyse the effect of bacterial infection on the survival, fecundity and behaviour of $T$. urticae spider mites are described in the Table S1 of the electronic supplementary material (Additional file 1).

To analyse the effect of bacteria on survival, oviposition and feeding behaviour of spider mites (models 1 to 10 ), the general procedure to build the statistical models was as follows: the treatment (bacterial concentration to which mites were exposed: LB only [i.e., OD0 for bacteria], OD1, OD10, OD25) was fit as a fixed explanatory variable, whereas blocks were fit as random explanatory variables. Survival data (models 1, 3, 5, 7) were analysed using a Cox proportional hazards mixed-effect model (coxme, kinship package). Hazard ratios were obtained as an estimate of the difference in survival rates (Crawley 2007) between the control (LB) and each of the other factor levels. As the oviposition data (number of eggs laid per female per day) were greatly overdispersed, they were analysed using a mixed model glmmadmb procedure (glmmADMB package). For these models (models 2, 4, 6, 8), error structures that best fit the errors distribution (e.g. zero-inflated quasi-poisson or negative binomial) were chosen based on AIC, the variable "day" was added as fixed explanatory variable and mite identity nested within blocks was fit as random explanatory variable across "days" to account for temporal autocorrelation across repeated measures on the same individuals (Crawley 2007). When a significant interaction was found between the variables "day" and "treatment", the effect of treatment was analysed within each day separately. The proportion of mites that fed (models 9 and 10) was computed using the function cbind (number of females fed vs. unfed) and subsequently analysed either with a mixed model glmmadmb procedure (glmmADMB package) with zero-inflated binomial 
error distribution to account for overdispersion (model 9), or with a generalized linear mixed effect model (glmer, Ime4 package) with a binomial error distribution (model 10). In all analyses, when the variable "treatment" was significant, a stepwise a posteriori procedure (Crawley 2007) to determine differences between treatments was carried out by aggregating factor levels together and by testing the fit of the simplified model using a likelihood ratio test (LRT), which is approximately distributed as a $\chi^{2}$ distribution (Bolker 2008).

To analyse the effect of olfactory cues on spider-mite avoidance of contaminated food (model 11), the time spent by each female on each side of the arena was computed using the function cbind (time spent on the side of the droplet containing bacteria or no droplet vs. time spent on the side of the droplet containing (B), and subsequently analysed using a generalized linear model ( $\mathrm{glm}$ ) using a quasibinomial error distribution to account for over-dispersion. The type of test (LB vs no droplet, a droplet with E. coli, or with P. putida) was fit as fixed explanatory variable along with the side (left or right) on which the control (LB) droplet was located. To determine whether, in each treatment, the mites spent significantly more time in one of the sides of the arena, the intercept of the model was changed to zero, which gives (in a model with categorical factors and a binomial distribution) the estimates of each fixed factor against a probability of 0.5 (Crawley 2007).

To analyse the effect of olfactory cues on spider-mite avoidance behaviour using the olfactometer (model 12), the type of test (LB vs E. coli or P. putida on cotton or on the entire plant) was fit as fixed explanatory variable, whereas block and the side (left or right) on which the control (LB) was located were fit as random explanatory variables. The olfactometer arm chosen by mites, a binary response variable (0: LB 1: bacteria) was then analysed using a generalized linear mixed effect model (glmer, Ime4 package) with a binomial error distribution. As before, the intercept of the model was changed to zero to determine whether the probability of a mite choosing one arm of the olfactometer differs significantly from 0.5 .

For all analyses, maximal models were simplified by sequentially eliminating nonsignificant terms to establish a minimal model (Crawley 2007), and the significance of the explanatory variables was established using $\chi^{2}$-tests or F-tests to account for over-dispersion (Bolker 2008). The significant values given in the text are for the minimal model, while nonsignificant values are those obtained before removing the variable from the model (Crawley 2007). Full datasets are given in Additional files 2 to 7. 


\section{RESULTS}

\section{Effects of bacteria spraying on spider-mite mortality and fecundity}

Spraying of $T$. urticae females with E. coli negatively affected their survival (treatment effect: $X^{2}{ }_{3}=80.21, P<0.0001$; model 1 ; Figure $1 \mathrm{~A}$ ). Contrast analyses revealed that mortality of mites sprayed with bacteria at OD10 did not increase compared to those sprayed with a concentration of OD1 (contrasts OD1 vs. OD10: $X^{2}{ }_{1}=1.01, P=0.32$ ). However, mortality caused by these treatments was 8.6-fold higher than that of mites sprayed with LB only (contrasts OD1-OD10 vs. LB: $X^{2}{ }_{1}=104.02, P<0.0001$ ), and 4.12-fold lower than that of mites sprayed with $E$. coli at OD25 (contrasts OD1-OD10 vs. OD25: $X_{1}{ }_{1}=9.13, P=0.003$ ). Spider mite oviposition increased similarly through time in all treatments (day*treatment interaction: $X^{2}{ }_{3}=6.82, P=0.08$; Main effect of day: $X_{1}^{2}=15.76, P<0.0001$; model 2; Figure 1B) but differ significantly between treatments (Main effect of treatment: $X^{2}{ }_{3}=35.80, P<$ 0.0001; model 2; Figure 1B). Indeed, contrast analyses showed that, although E. coli at OD25 did not significantly affect oviposition (contrasts LB vs. OD25: $X^{2}{ }_{1}=1.40, P=0.24$ ), females laid more eggs per day after being sprayed with E. coli at OD1 or OD10 (contrasts OD1 vs. OD10: $X^{2}=0.14, P=0.71$; LB-OD25 vs. OD1-OD10: $X^{2}{ }_{1}=34.36, P<0.0001$ ).

Similarly to E. coli, spraying of P. putida decreased female survival (main effect of treatment: $X^{2}{ }_{3}=138.19, P<0.0001$; model 3; Figure $1 \mathrm{C}$ ) to the same extent for all bacterial concentration used (contrasts OD1 vs. OD10 vs. OD25: $X_{2}^{2}=2.58, P=0.28$ ). Contrasting with the results for $E$. coli, the effect of $P$. putida on spider-mite oviposition varied with the number of days after spraying (day*treatment interaction: $X^{2}{ }_{3}=8.12, P=0.04$; model 4 ; Figure 1D). Indeed, the separate analyses of each day following spraying revealed that $P$. putida decreased significantly the oviposition of spider mites during the first two days

following spraying (main effect of treatment at day $1: X^{2}{ }_{3}=15.10, P=0.002$, and at day $2: X^{2}{ }_{3}$ $=9.51, P=0.02$ ). During the first day females sprayed with OD10 or OD25 (contrasts OD10 vs. OD25: $X^{2}{ }_{1}=1.66, P=0.20$ ) laid fewer eggs than those sprayed with LB or OD1 (contrasts LB vs. OD1: $X^{2}{ }_{1}=0.31, P=0.58$; LB-OD1 vs. OD10-OD25: $X^{2}{ }_{1}=13.14, P<0.001$ ), while during the second day all bacterial doses led to decreased oviposition compared to the control LB to a similar extent (contrasts OD1 vs. OD10 vs. OD25: $X^{2}{ }_{2}=0.18, P=0.91$ ). In contrast, on the following two days no effect on oviposition could be detected (main effect of treatment at 
day 3: $X^{2}{ }_{3}=0.91, P=0.82$, and at day $\left.4: X^{2}{ }_{3}=3.97, P=0.28\right)$. Note, however, that for unknown reasons, oviposition was extremely low in the $P$. putida spraying experiment (compare Figures 1B and 1D).

A

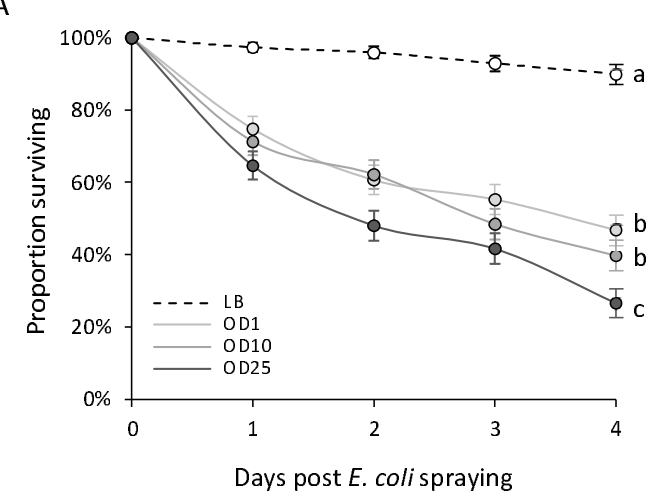

B

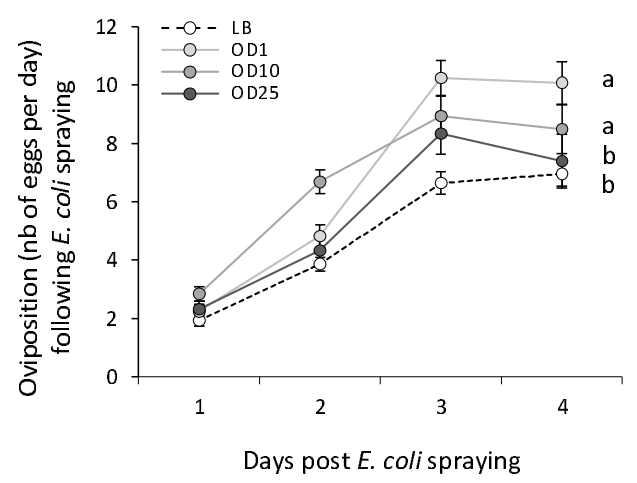

C

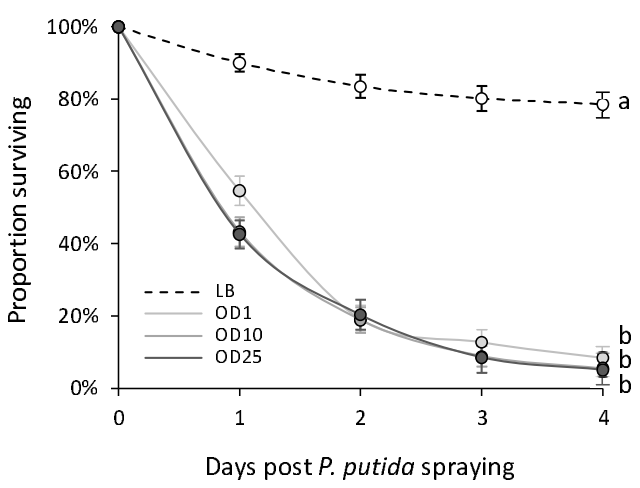

D

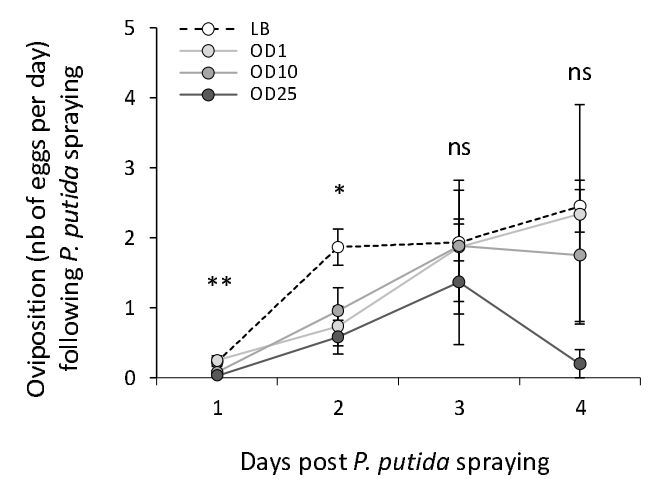

Figure 1. Effect of bacteria spraying on spider-mite survival $(A, C)$ and daily oviposition (B, D). Females were exposed to different concentrations of either E. coli (A, B) or P. putida (C, D). In (B) and (D), points represent the mean number of eggs ( \pm s.e.) for each experimental day. In (A), (B) and (C), identical letter superscripts indicate non-significant differences between treatments at the $5 \%$ level (a posteriori contrasts). In (D), as a significant interaction between day and treatment was found, superscripts indicate the significance of the treatment effect for each day (ns: no significant differences at the $5 \%$ level, ${ }^{*} p<0.05, * *$ $\mathrm{p}<0.01$ ). White dots and black dashed line: LB; Light grey dots and lines: OD1; Medium grey dots and lines: OD10; Dark grey points and lines: OD25.

\section{Effects of bacteria ingestion on spider-mite mortality and oviposition}

Feeding on E. coli increased the mortality of $T$. urticae by 4-fold (main effect of treatment: $X^{2}{ }_{3}=10.36, P=0.02$; model 5; Figure $2 \mathrm{~A}$ ), independently of the ingested bacterial concentration (contrasts OD1 vs. OD10 vs. OD25: $X^{2}{ }_{2}=1.56, P=0.46$ ). The effect of $E$. coli ingestion on spider-mite oviposition varied with the number of days after feeding 
(day*treatment interaction: $X^{2}{ }_{3}=8.10, P=0.04$; model 6; Figure 2B). Indeed, spider mite oviposition differed between treatments at days 1 and 2 (main effect of treatment at day 1 : $X^{2}{ }_{3}=17.40, P<0.001$, and at day $2: X^{2}{ }_{3}=12.85, P=0.005$ ) but not at days 3 and 4 (main effect of treatment at day $3: X^{2}{ }_{3}=3.90, P=0.27$, and at day $4: X^{2}{ }_{3}=2.08, P=0.56$ ). For both day 1 and day 2, the females fed on OD10 and OD25 had a decreased oviposition (contrasts OD10 vs. OD25 for day $1: X_{1}{ }_{1}=1.74, P=0.19$, and for day $2: X_{1}{ }_{1}=0.75, P=0.39$ ) while those fed on OD1 had the same oviposition than those fed on LB only (contrasts LB vs. OD1 for day 1: $X^{2}{ }_{1}=0.21, P=0.66$, and for day $2: X^{2}{ }_{1}=0.04, P=0.84$; contrasts LB-OD1 vs. OD10-OD25 for day $1: X_{1}^{2}=15.49, P<0.0001$, and for day $\left.2: X_{1}{ }_{1}=12.06, P=0.001\right)$.

A

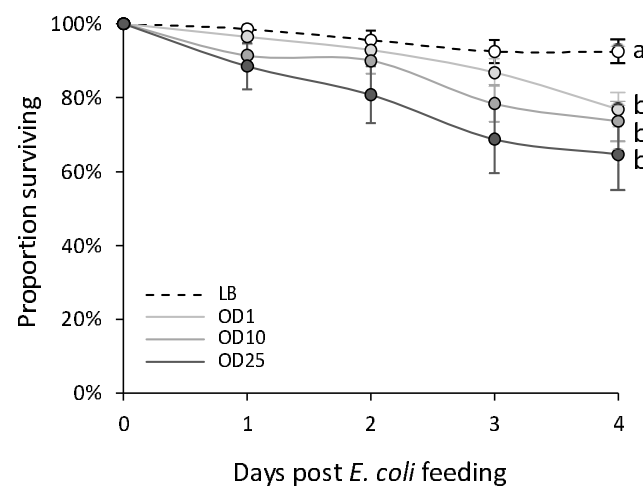

B

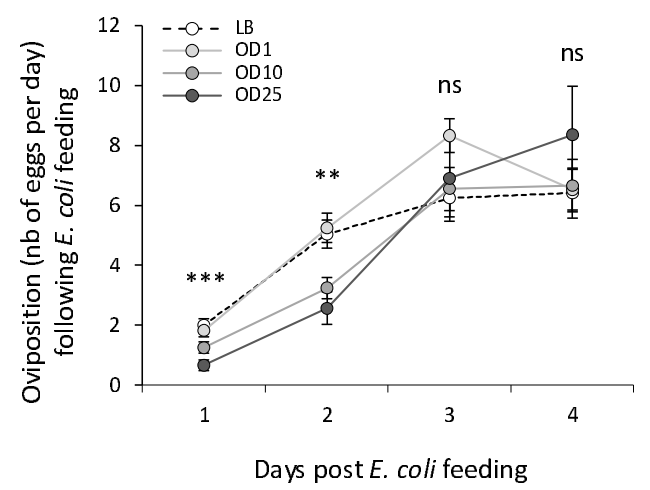

C

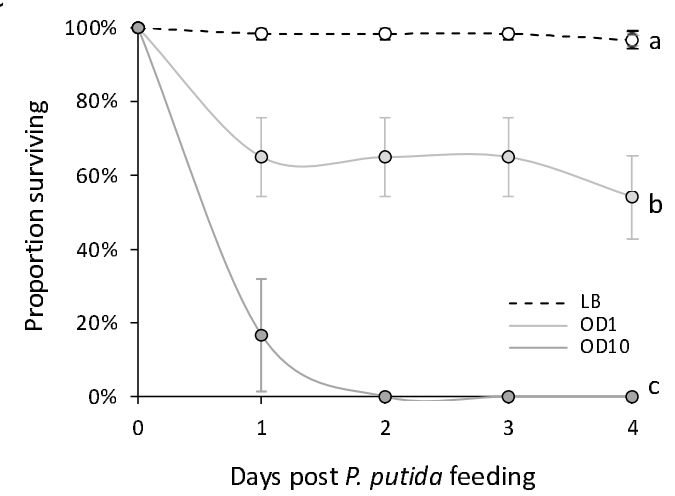

D

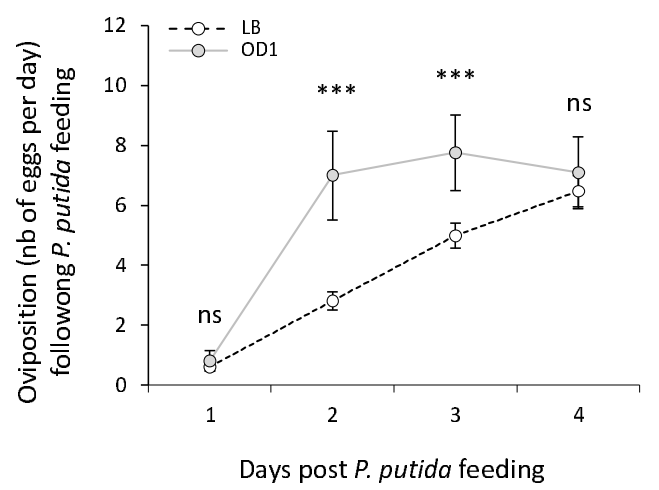

Figure 2. Effect of bacteria ingestion on spider-mite survival $(A, C)$ and daily oviposition (B, D). Females were exposed to different concentrations of either E. coli (A, B) or P. putida (C, D). In (B) and (D), points represent mean number of eggs ( \pm s.e.) for each day experimental day. In $(A)$ and $(C)$, identical letter superscripts indicate non-significant differences between treatments at the $5 \%$ level (a posteriori contrasts). In (B) and (D), as significant interactions between day and treatment were found, superscripts indicate the significance of the treatment effect for each day (ns: no significant differences at the $5 \%$ level, $* * p<0.01$, $* * * p<0.001)$. White points and black dashed line: LB; Light grey dots and lines: OD1; Medium grey dots and lines: OD10; Dark grey dots and lines: OD25. 
As only one mite fed on food contaminated with P. putida at OD25 (see below), we could not study the effect of ingestion at this concentration on survival. For the remaining concentrations, feeding on $P$. putida affected spider mite mortality (main effect of treatment: $X^{2}{ }_{3}=23.30, P<0.0001$; model 7; Figure $2 \mathrm{C}$ ). Mortality was higher when mites fed on food containing $P$. putida at OD10 than at OD1 (contrasts OD1 vs. OD10: $X^{2}{ }_{1}=4.22, P=$ 0.04), and feeding on bacteria at both concentrations increased spider-mite mortality comparing to control mites (contrasts OD1 vs. LB: $X^{2}{ }_{1}=19.58, P<0.0001$ ). Since no mites survived after ingestion of $P$. putida at OD10, only the effect of OD1 on female oviposition could be studied (Figure $2 \mathrm{C}$ ), and the statistical analyses revealed that this effect varied through time (day*treatment interaction: $X^{2}{ }_{1}=6.78, P=0.009$; model 8; Figure 2D). While all mites laid the same number of eggs at days 1 and 4 (main effect of treatment at day $1: X^{2}{ }_{1}=$ $0.39, P=0.53$, and at day $\left.4: X^{2}=0.11, P=0.74\right)$, at days 2 and 3 the ingestion of $P$. putida at OD1 increased the number of eggs laid compared to the control (main effect of treatment at day $2: X_{1}^{2}=30.34, P<0.0001$, and at day $\left.3: X^{2}{ }_{1}=11.01, P<0.001\right)$.

\section{Avoidance of bacteria ingestion}

Fewer mites fed on food with $E$. coli than on food with LB only (main effect of treatment: $F_{3,34}=20.13, P<0.0001$; model 9; Figure 3A). This effect, however, did not increase with the bacterial concentration, as the proportion of mites avoiding contaminated food was the lowest at OD10 (contrasts LB vs. OD10: $X^{2}{ }_{1}=3.85, P<0.05$ ), intermediate at OD1 (contrasts OD1 vs OD10: $X^{2}{ }_{1}=4.61, P=0.03$ ), and the highest at OD25 (contrasts OD1 vs OD25: $X^{2}{ }_{1}=$ $6.69, P=0.01)$.

Similarly, fewer mites fed on LB with $P$. putida than on LB only (main effect of treatment: $X^{2}{ }_{3}=138.57, P<0.0001$; model 10; Figure 3B). Indeed, $43 \%$ of the mites fed on LB, but only $12 \%$ fed on food containing $P$. putida at OD1 (contrasts LB vs OD1: $X^{2}{ }_{1}=38.62, P$ $<0.0001$ ), and only about $1 \%$ fed on food with P. putida at OD10 or OD25 (contrasts OD10 vs OD25: $X_{1}^{2}=2.71, P=0.10$; contrasts OD1 vs OD10-25: $X_{1}^{2}=21.87, P<0.0001$ ). 

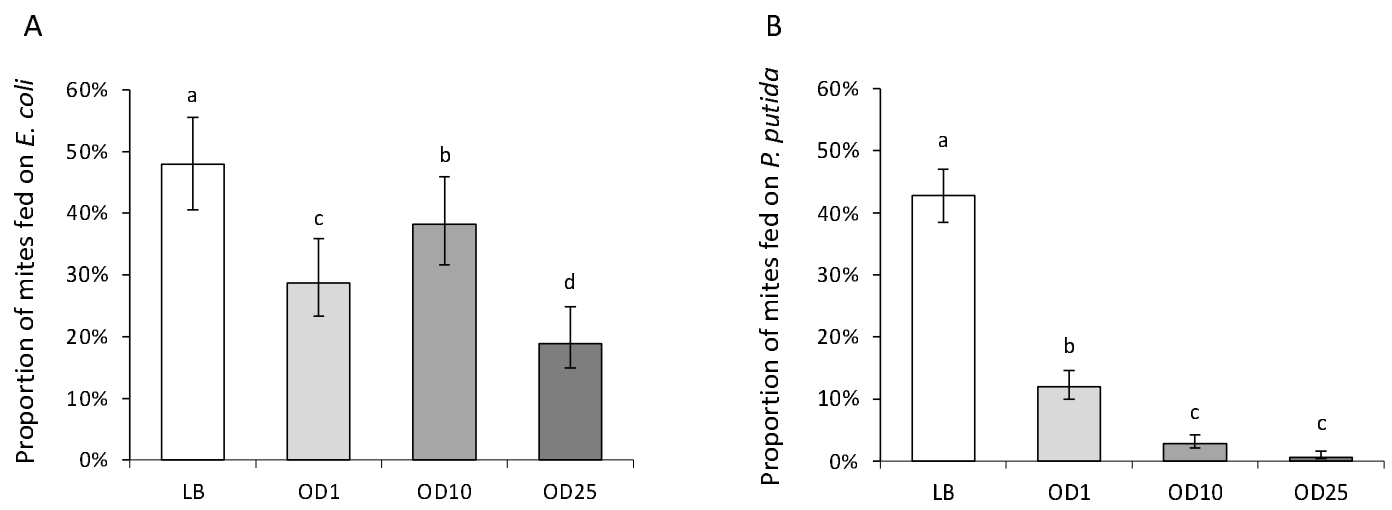

Figure 3. Avoidance of bacteria ingestion by $T$. urticae females. Bars and dots represent mean ( \pm s.e.) proportion of females feeding on E. coli (A) or P. putida (B). Estimates were obtained from the GLMM statistical model that takes into account variation among replicates and blocks as random effect, and corrects for zero-inflation in the case of feeding on E. coli. Standard errors were obtained from the upper and lower confidence intervals given by the model. Identical superscripts indicate non-significant differences between treatments at the $5 \%$ level (a posteriori contrasts). White bars: LB; Light grey bars: OD1; Medium grey bars: OD10; Dark grey bars: OD25.

\section{Effects of volatile cues on spider-mite avoidance behaviour}

When given the choice between leaf discs containing LB with bacteria or LB alone, spider mites showed no preference (main effect of the type of test: $F_{2,63}=1.01, P=0.37$; model 11 ; Figure 4A). Indeed, the time spent by females on each side of the arena was the same, irrespective of the type of choice presented to the mites (no droplet vs. LB: $t(1)=-1.54, P=$ $0.13 ;$ E. coli vs. $\mathrm{LB}: \mathrm{t}(1)=0.06, P=0.95 ; P$. putida vs. $\mathrm{LB}: \mathrm{t}(1)=0.25, P=0.81$ ). Moreover, in all types of tests performed with the Y-maze olfactometer (main effect of type of test: $X^{2}{ }_{3}=$ 3.86, $P=0.28$; model 12) mites did not show any preference for the arm of the olfactometer free from bacteria (difference to 0.5 for E. coli vs LB on cotton: $z=-1.12, \mathrm{P}=0.27 ;$. putida vs LB on cotton: $\mathrm{z}=-0.89, \mathrm{P}=0.37 ;$ E. coli vs $\mathrm{LB}$ on plant: $\mathrm{z}=0.22, \mathrm{P}=0.82 ; P$. putida vs $\mathrm{LB}$ on plant: $z=1.18, P=0.18$; Figure $4 B$ ). Thus, we found no evidence for the effect of volatile cues on spider-mite avoidance behavior toward bacteria. 
A

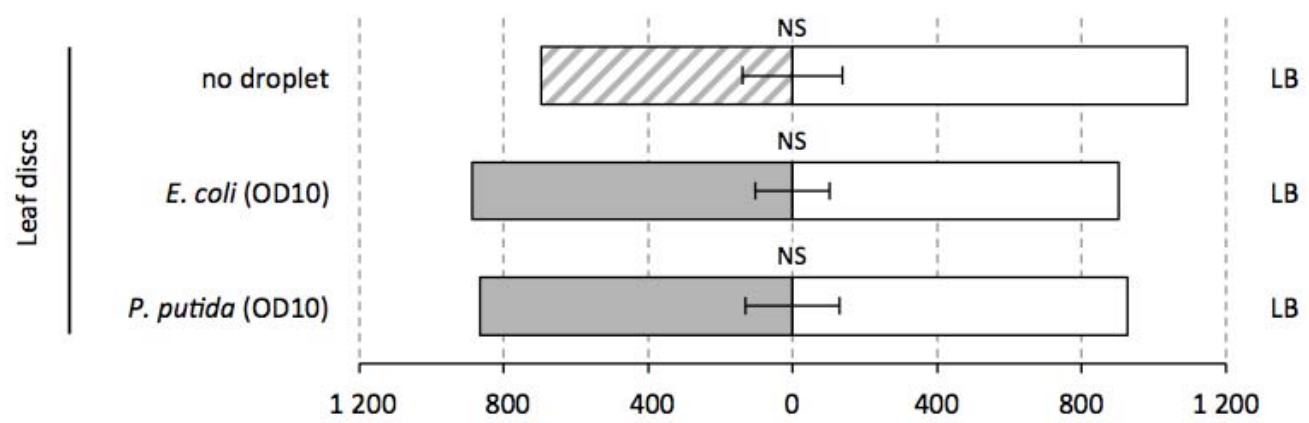

Time (seconds) spent in each side of the arena

B

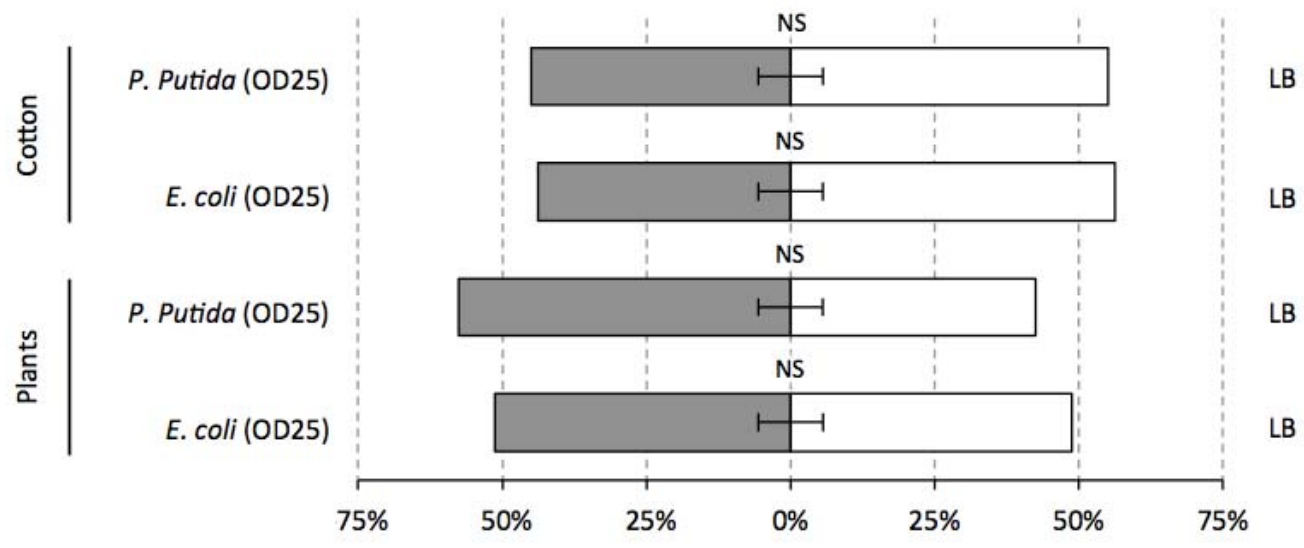

Proportion of mites choosing each arm of the olfactometer

Figure 4. Effect of volatile cues on spider-mite avoidance behaviour. Bars represent mean $( \pm$ s.e.) time spent by females close to a leaf disc free with either no droplet, or a droplet containing either LB or bacteria (E. coli or P. putida) at OD10 (A), and the proportion of females choosing an arm of the olfactometer containing either bacteria (E. coli or P. putida) at OD25 or LB, on cotton or on entire bean plants (B). NS indicates that no significant differences have been found at the $5 \%$ level. White bars: LB; Light grey bars: OD1; Medium grey bars: OD10; Dark grey bars: OD25; Dashed bars: absence of droplet. 


\section{DISCUSSION}

In this study, we found that spider mites die upon infection with Escherichia coli and Pseudomonas putida both by spraying and ingestion. Moreover, we show that they avoid contaminated food and that odours are probably not involved in such discrimination.

In a previous study, we have shown that mites do not mount an effective immune response towards systemic bacterial infections (Santos-Matos et al. 2017). This result was intriguing, as mortality upon infection was very high, indicating that bacteria impose severe fitness costs to spider mites, which should select for traits that allow avoiding such costs. Hence, we here tested for the generality of these costs for other putative infection routes, and for the presence of other potential defence mechanisms, namely behavioural. We found that spraying mites with bacteria also led to significant mortality, which suggests that bacteria gained access to the body cavity of the mites. This may have occurred by ingestion (as the sprayed bacteria remain in the leaves, hence mites may feed upon them), but most likely also through the cuticle (e.g. via spiracles or tracheae). Both cuticle and gut epithelium may thus be permeable to bacterial infections. We then tested whether ingestion alone would be sufficient to induce severe mortality. We found this to be case. However, mortality levels induced by feeding alone were less severe than those observed with spraying (see Figures $1 \mathrm{~A}$ and $\mathrm{C}$ and $2 \mathrm{~A}$ and $\mathrm{C}$ ), which can be due to differences between protocols, but also to additional mortality induced by bacteria penetrating the cuticle. Indeed, spraying may lead to a higher amount of bacteria per mite than feeding on contaminated food. Testing this hypothesis would, however, require data on the bacterial loads per mite after infection. Nevertheless, the fact that injection with $E$. coli led to $100 \%$ mortality even at the lowest dose tested (Santos-Matos et al. 2017) whereas, in this study, some mites survived following bacterial exposure through other routes, suggests that cuticular and epithelial barriers confer some degree of protection. Importantly, these barriers may be much more effective in nature than in our experimental conditions where the amount of bacteria used is extremely high. However, some degree of permeability and ensuing exposure to bacterial infections are likely to occur in nature and, as discussed below, may select for spider-mite behavioural strategies to avoid exposure to pathogens.

We observed different responses in oviposition upon infection. Overall, oral infection of spider mites with $E$. coli resulted in decreased oviposition rate, whereas spraying led to an 
increase of this trait. Conversely, oral infection with $P$. putida resulted in increased oviposition whereas spraying led to a reduction of this trait. An interesting pattern thus seems to emerge between virulence and oviposition, whereby low virulence entails lower fecundity and extremely virulent infections induce the opposite response (i.e. increased oviposition). Often, infection entails a cost in terms of oviposition reduction, as observed in many species, such as mosquitos (Pigeault et al. 2015; Scholte et al. 2006; Zele et al. 2018) or sticklebacks (Heins 2012). However, some hosts are able to compensate infection-driven fitness costs by changing the timing of their reproductive efforts (i.e. 'fecundity compensation'; Parker et al. 2011; Vezilier et al. 2015). For example, Daphnia magna produces more offspring early in life when exposed to the microsporidian parasite Glugoides intestinalis (Chadwick and Little 2005). Our data suggest that the effects of bacterial infection on oviposition include both effects described above, with cost or compensation being contingent upon bacterial species and infection mode, as shown in other systems (Martins et al. 2013). More information on infection dynamics in spider mites, the relationship between total reproductive output and longevity, as well as how it affects other life-history traits, such as egg hatching, will help clarify this issue.

Independently of the route of infection tested, $E$. coli was less pathogenic than $P$. putida. Although a same bacterial density measured at $\mathrm{OD}_{600}$ does not correspond to the same number of bacteria for each species, the increased pathogenicity of $P$. putida cannot be due to an increased concentration compared to $E$. coli (i.e. a lower number of $P$. putida than of $E$. coli result in the same OD). Moreover, this result is consistent with previous work using systemic infection in Drosophila (Martins et al. 2013), and thus generalizes the virulence hierarchy between these bacteria beyond the infection route. E. coli is a particularly mild pathogen compared to P. putida.

We found that the avoidance response regarding $P$. putida ingestion was stronger than that observed for $E$ coli. Possibly, spider mites detect $E$. coli to a lesser extent than they detect $P$. putida, and/or the avoidance level depends on the magnitude of the threat. In our study, mites showed higher avoidance in feeding on P. putida, which is more pathogenic than E. coli. This would suggest both that bacterial avoidance is costly (see Buck et al. 2018) and consequently selected only for highly pathogenic bacteria, and that spider mites are able to discriminate between different degrees of pathogenicity. Within bacterial species, mites avoided more often E. coli at high concentrations and less at low concentrations, but 
were equally affected by all concentrations tested. This may entail that, under natural conditions, low E. coli concentrations will lead to higher mortality within populations. However, this was not the case for P. putida, as high concentrations of this bacterium induced a higher mortality and were more avoided by spider mites than low concentrations. The avoidance to feed on contaminated food is not necessarily triggered by an avoidance of infection per se. Indeed, mites may also use bacteria as a proxy for low-quality food, such as rotten food (Janzen 1977). Conversely, in absence of a direct detection of pathogens, hosts may use rotten food as a cue that is reliably associated with pathogens to avoid becoming infected (see Buck et al. 2018). Thus, the feeding avoidance observed in our experiment may reflect a more general response against infection by diverse pathogens.

We found that avoidance was not triggered by olfactory cues, either directly produced by the bacteria or resulting from their interaction with the plants. We cannot exclude the possibility that these results are due to limitations in our experimental setup. However, both set-ups have been previously used to show the ability of spider mites to detect olfactory cues, albeit in other contexts (Fouks and Lattorff 2011; Pallini et al. 1997; Parker et al. 2010; Rodrigues et al. 2017; Rondot and Reineke 2017; Tasin et al. 2012). Nevertheless, our results suggest that avoidance happens locally, in the plant, and not at long distance, and are consistent with spider mites not perceiving bacteria odours but, instead, avoiding rotten food after tasting. Indeed, gustatory avoidance has been previously shown for other arthropods, such as Drosophila avoiding bacterial LPS through the activation of the protein TRPA (Soldano et al. 2016).

Gustatory avoidance is likely to have strong effects locally, both in terms of host mortality and parasite transmission. The survival of spider mites is drastically reduced when bacteria penetrate their body as they have a deficient immune response upon infection (Santos-Matos et al. 2017). Moreover, bacteria proliferate inside their body (Santos-Matos et al. 2017), which can increase the likelihood of transmission (Anderson and May 1979). Consequently, individuals with reduced avoidance are more likely to be exposed and, thus, to die upon bacterial infection. Natural populations of spider mites are frequently at highdensity of individuals, which favours pathogen transmission (Grbic et al. 2011). If individuals do not avoid pathogens and bacteria proliferate within hosts, this might lead to an epidemic and, in an extreme case, to population extinction. 
Our results show that spider mites, while not mounting an effective immune response upon infection by bacteria, have alternative defensive strategies. These include the cuticle and gut barriers of the body, apparent fecundity compensation following infection, and behavioural avoidance of contaminated food sources. Therefore, these data highlights the importance of investigating different host defensive strategies in order to fully analyse the outcome of pathogen infection. Moreover, our results highlight the need of further research to determine the relevance of such findings in nature.

\section{ACKNOWLEDGEMENTS}

We thank Miodrag Grbic for the "bubble machine" and José Feijó for the air pump, both of which were necessary to make the parafilm bubbles (feeding experiments). We thank Arne Jansen and Luísa Vasconcelos for the material needed to make the $Y$ olfactometer, and Francisco Dionísio for providing access to his laboratory, material and reagents for bacterial work. We also thank members of the Sucena and Magalhães labs for useful discussions and suggestions. This work was funded by an FCT-Tubitak agreement (FCT-TUBITAK/0001/2014) to SM and Ibrahim Cakmak. FZ was funded through an FCT Post-Doc fellowship (SFRH/BPD/125020/2016). Funding agencies did not participate in the design or analysis of experiments. We declare that we do not have any conflict of interest. 


\section{REFERENCES}

Aksoy H, Ozman-Sullivan S, Ocal H, Celik N, Sullivan G (2008) The effects of Pseudomonas putida biotype B on Tetranychus urticae (Acari: Tetranychidae). Exp. Appl. Acarol. 46:223-230. doi: 10.1007/s10493-008-9155-9

Altincicek B, Gross J, Vilcinskas A (2008) Wounding-mediated gene expression and accelerated viviparous reproduction of the pea aphid Acyrthosiphon pisum. Insect Mol. Biol. 17:711-716. doi: 10.1111/j.1365-2583.2008.00835.x

Anderson RM, May RM (1979) Population biology of infectious diseases: Part 1. Nature 280:361-367. doi: 10.1038/280361a0

Ateyyat MA, Shatnawi M, Al-Mazra'awi M (2010) Isolation and identification of culturable forms of bacteria from the sweet potato whitefly Bemesia tabaci Genn. (Homoptera: Aleyrodidae) in Jordan. Turkish Journal of Agriculture and Forestry 34:225-234. doi: 10.3906/tar-0902-35

Ayres JS, Schneider DS (2008) A signaling protease required for melanization in Drosophila affects resistance and tolerance of infections. PLoS Biol. 6:2764-2773. doi: 10.1371/journal.pbio.0060305

Bodenhausen N, Horton MW, Bergelson J (2013) Bacterial communities associated with the leaves and the roots of Arabidopsis thaliana. PLoS One 8. doi: 10.1371/journal.pone.0056329

Bolker BM (2008) Ecological models and data in R Princeton University Press, New Jersey Bouwman KM, Hawley DM (2010) Sickness behaviour acting as an evolutionary trap? Male house finches preferentially feed near diseased conspecifics. Biol. Lett. 6:462-465. doi: $10.1098 / \mathrm{rsbl} .2010 .0020$

Broadbent AB, Matteoni JA (1990) Acquisition and transmission of Pseudomonas cichorii by Liriomyza trifolii (Diptera, Agromyzidae). Proceedings of the Entomological Society of Ontario 121:79-84

Bucher GE, Stephens JM (1957) A disease of grasshoppers caused by the bacterium Pseudomonas aeruginosa (Schroeter) Migula. Canadian Journal of Microbiology 3:611-625. doi: 10.1139/m57-067

Buck JC, Weinstein SB, Young HS (2018) Ecological and evolutionary consequences of parasite avoidance. Trends Ecol. Evol. 33:619-632. doi: 10.1016/j.tree.2018.05.001 
Chadwick W, Little TJ (2005) A parasite-mediated life-history shift in Daphnia magna. Proc. R. Soc. Lond. B 272:505-509. doi: 10.1098/rspb.2004.2959

Clayton DH (1991) The influence of parasites on host sexual selection. Parasitology Today 7:329-334. doi: 10.1016/0169-4758(91)90211-6

Clemente SH, Rodrigues LR, Ponce R, Varela SAM, Magalhães S (2016) Incomplete species recognition entails few costs in spider mites, despite first-male precedence. Behav. Ecol. Sociobiol. 70:1161-1170. doi: 10.1007/s00265-016-2124-0

Commare RR et al. (2002) Pseudomonas fluorescens based bio-formulation for the management of sheath blight disease and leaffolder insect in rice. Crop Protection 21:671-677. doi: 10.1016/S0261-2194(02)00020-0

Cornet S, Nicot A, Rivero A, Gandon S (2013) Malaria infection increases bird attractiveness to uninfected mosquitoes. Ecol. Lett.:323-329. doi: 10.1111/ele.12041

Crawley MJ (2007) The R Book John Wiley \& Sons, Ltd, Chichester, England

Cremer S, Pull CD, Furst MA (2018) Social immunity: emergence and evolution of colonylevel disease protection. In: Berenbaum MR (ed) Annual Review of Entomology, Vol 63, vol 63, pp 105-123

Curtis VA (2014) Infection-avoidance behaviour in humans and other animals. Trends in Immunology 35:457-464. doi: 10.1016/j.it.2014.08.006

Eakin L, Wang M, Dwyer G (2015) The effects of the avoidance of infectious hosts on infection risk in an insect-pathogen interaction. Am. Nat. 185:100-112. doi: $10.1086 / 678989$

Fill A, Long EY, Finke DL (2012) Non-consumptive effects of a natural enemy on a non-prey herbivore population. Ecol. Entomol. 37:43-50. doi: 10.1111/j.13652311.2011.01333.x

Fouks B, Lattorff HMG (2011) Recognition and avoidance of contaminated flowers by foraging bumblebees (Bombus terrestris). PLoS One 6. doi: 10.1371/journal.pone.0026328

Gerardo NM et al. (2010) Immunity and other defenses in pea aphids, Acyrthosiphon pisum. Genome Biology 11. doi: 10.1186/gb-2010-11-2-r21

Grbic M et al. (2011) The genome of Tetranychus urticae reveals herbivorous pest adaptations. Nature 479:487-492. doi: 10.1038/nature10640 
Hall MD, Bento G, Ebert D (2017) The evolutionary consequences of stepwise infection processes. Trends Ecol. Evol. 32:612-623. doi: 10.1016/j.tree.2017.05.009

Hart BL (1990) Behavioral adaptations to pathogens and parasites - 5 strategies. Neuroscience and Biobehavioral Reviews 14:273-294. doi: 10.1016/s01497634(05)80038-7

Hart BL (1994) Behavioral defense against parasites - Interaction with parasite invasiveness. Parasitology 109:S139-S151. doi: 10.1017/s0031182000085140

Heins DC (2012) Fecundity compensation in the three-spined stickleback Gasterosteus aculeatus infected by the diphyllobothriidean cestode Schistocephalus solidus. Biological Journal of the Linnean Society 106:807-819. doi: 10.1111/j.10958312.2012.01907.x

Janzen DH (1977) Why fruits rot, seeds mold, and meat spoils. Am. Nat. 111:691-713. doi: $10.1086 / 283200$

Karvonen A, Seppala O, Valtonen ET (2004) Parasite resistance and avoidance behaviour in preventing eye fluke infections in fish. Parasitology 129:159-164. doi: $10.1017 / \mathrm{s} 0031182004005505$

Kiesecker JM, Skelly DK, Beard KH, Preisser E (1999) Behavioral reduction of infection risk. Proc. Natl Acad. Sci. USA 96:9165-9168. doi: 10.1073/pnas.96.16.9165

Kim DJ, Chung SG, Lee SH, Choi JW (2012) Relation of microbial biomass to counting units for Pseudomonas aeruginosa. African Journal of Microbiology Research 6:4620-4622. doi: $10.5897 /$ ajmr10.902

Kuraishi T, Binggeli O, Opota O, Buchon N, Lemaitre B (2011) Genetic evidence for a protective role of the peritrophic matrix against intestinal bacterial infection in Drosophila melanogaster. Proc. Natl Acad. Sci. USA 108:15966-15971. doi: 10.1073/pnas.1105994108

Laughton AM, Garcia JR, Gerardo NM (2016) Condition-dependent alteration of cellular immunity by secondary symbionts in the pea aphid, Acyrthosiphon pisum. Journal of Insect Physiology 86:17-24. doi: 10.1016/j.jinsphys.2015.12.005

Lemaitre B, Nicolas E, Michaut L, Reichhart JM, Hoffmann JA (1996) The dorsoventral regulatory gene cassette spatzle/Toll/cactus controls the potent antifungal response in Drosophila adults. Cell 86:973-983. doi: 10.1016/s0092-8674(00)80172-5 
Liu XY, Sun YL, Korner CJ, Du XR, Vollmer ME, Pajerowska-Mukhtar KM (2015) Bacterial leaf infiltration assay for fine characterization of plant defense responses using the Arabidopsis thaliana-Pseudomonas syringae pathosystem. Jove-Journal of Visualized Experiments. doi: $10.3791 / 53364$

Martins NE, Faria VG, Teixeira L, Magalhaes S, Sucena E (2013) Host adaptation is contingent upon the infection route taken by pathogens. PLoS Pathog. 9. doi: 10.1371/journal.ppat.1003601

Meric G, Kemsley EK, Falush D, Saggers EJ, Lucchini S (2013) Phylogenetic distribution of traits associated with plant colonization in Escherichia coli. Environ. Microbiol. 15:487-501. doi: 10.1111/j.1462-2920.2012.02852.x

Mnyone LL, Koenraadt CJM, Lyimo IN, Mpingwa MW, Takken W, Russell TL (2010) Anopheline and culicine mosquitoes are not repelled by surfaces treated with the entomopathogenic fungi Metarhizium anisopliae and Beauveria bassiana. Parasit Vectors 3. doi: 10.1186/1756-3305-3-80

Moore J (2002) Parasites and the behaviour of animals, 1st edition edn. Oxford University Press, Oxford

Ormond EL, Thomas APM, Pell JK, Freeman SN, Roy HE (2011) Avoidance of a generalist entomopathogenic fungus by the ladybird, Coccinella septempunctata. Fems Microbiol. Ecol. 77:229-237. doi: 10.1111/j.1574-6941.2011.01100.x

Pallini A, Janssen A, Sabelis MW (1997) Odour-mediated responses of phytophagous mites to conspecific and heterospecific competitors. Oecologia 110:179-185. doi: $10.1007 / \mathrm{s} 004420050147$

Parker BJ, Barribeau SM, Laughton AM, de Roode JC, Gerardo NM (2011) Non-immunological defense in an evolutionary framework. Trends Ecol. Evol. 26:242-248. doi: 10.1016/j.tree.2011.02.005

Parker BJ, Barribeau SM, Laughton AM, Griffin LH, Gerardo NM (2017) Life-history strategy determines constraints on immune function. Journal of Animal Ecology 86:473-483. doi: $10.1111 / 1365-2656.12657$

Parker BJ, Elderd BD, Dwyer G (2010) Host behaviour and exposure risk in an insectpathogen interaction. Journal of Animal Ecology 79:863-870. doi: 10.1111/j.13652656.2010.01690.x 
Pigeault R et al. (2015) Avian malaria: a new lease of life for an old experimental model to study the evolutionary ecology of Plasmodium. Philos. Trans. R. Soc. B Biol. Sci. 370. doi: $10.1098 /$ rstb.2014.0300

Poinar G, Poinar R (1998) Parasites and pathogens of mites. Annu. Rev. Entomol. 43:449-469. doi: 10.1146/annurev.ento.43.1.449

Rastogi G, Sbodio A, Tech JJ, Suslow TV, Coaker GL, Leveau JHJ (2012) Leaf microbiota in an agroecosystem: spatiotemporal variation in bacterial community composition on field-grown lettuce. ISME J 6:1812-1822. doi: 10.1038/ismej.2012.32

Rodrigues LR, Figueiredo ART, Varela SAM, Olivieri I, Magalhaes S (2017) Male spider mites use chemical cues, but not the female mating interval, to choose between mates. Exp. Appl. Acarol. 71:1-13. doi: 10.1007/s10493-016-0103-9

Rondot Y, Reineke A (2017) Association of Beauveria bassiana with grapevine plants deters adult black vine weevils, Otiorhynchus sulcatus. Biocontrol Science and Technology 27:811-820. doi: 10.1080/09583157.2017.1347604

Santos-Matos $\mathrm{G}$ et al. (2017) Tetranychus urticae mites do not mount an induced immune response against bacteria. Proc. R. Soc. B 284:8. doi: 10.1098/rspb.2017.0401

Schneider M, Dorn A (2001) Differential infectivity of two Pseudomonas species and the immune response in the milkweed bug, Oncopeltus fasciatus (Insecta : Hemiptera). J. Invertebr. Pathol. 78:135-140. doi: 10.1006/jipa.2001.5054

Scholte EJ, Knols BGJ, Takken W (2006) Infection of the malaria mosquito Anopheles gambiae with the entomopathogenic fungus Metarhizium anisopliae reduces blood feeding and fecundity. J. Invertebr. Pathol. 91:43-49. doi: 10.1016/j.jip.2005.10.006

Seo S, Matthews KR (2012) Influence of the plant defense response to Escherichia coli 0157:H7 Cell surface structures on survival of that enteric pathogen on plant surfaces. Appl. Environ. Microbiol. 78:5882-5889. doi: 10.1128/aem.01095-12

Soldano A et al. (2016) Gustatory-mediated avoidance of bacterial lipopolysaccharides via TRPA1 activation in Drosophila. Elife 5. doi: 10.7554/eLife.13133

Solomon EB, Pang HJ, Matthews KR (2003) Persistence of Escherichia coli 0157 : H7 on lettuce plants following spray irrigation with contaminated water. Journal of Food Protection 66:2198-2202. doi: 10.4315/0362-028x-66.12.2198

Solomon EB, Yaron S, Matthews KR (2002) Transmission of Escherichia coli O157 : H7 from contaminated manure and irrigation water to lettuce plant tissue and its subsequent 
internalization. Appl. Environ. Microbiol. 68:397-400. doi: 10.1128/aem.68.1.397400.2002

Tasin M, Knudsen GK, Pertot I (2012) Smelling a diseased host: grapevine moth responses to healthy and fungus-infected grapes. Animal Behaviour 83:555-562. doi: 10.1016/j.anbehav.2011.12.003

Thomas GM, Poinar GO (1973) Report od diagnoses of diseased insects 1962-1972. Hilgardia 42:261-359. doi: 10.3733/hilg.v42n08p261

Vezilier J, Nicot A, Gandon S, Rivero A (2015) Plasmodium infection brings forward mosquito oviposition. Biol. Lett. 11. doi: 10.1098/rsbl.2014.0840

Vodovar $\mathrm{N}$ et al. (2006) Complete genome sequence of the entomopathogenic and metabolically versatile soil bacterium Pseudomonas entomophila. Nature Biotechnology 24:673-679. doi: 10.1038/nbt1212

Vodovar $\mathrm{N}$ et al. (2005) Drosophila host defense after oral infection by an entomopathogenic Pseudomonas species. Proc. Natl Acad. Sci. USA 102:11414-11419. doi: 10.1073/pnas.0502240102

Zele F, Denoyelle J, Duron O, Rivero A (2018) Can Wolbachia modulate the fecundity costs of Plasmodium in mosquitoes? Parasitology 145:775-782. doi: $10.1017 / \mathrm{s} 0031182017001330$ 